\title{
Global plasma demand in 2015
}

\section{Patrick Robert}

President, The Marketing Research Bureau, Inc., 284 Racebrook Road, Orange, CT 06477, USA Tel.: +1203799 0298; Fax: +12038918855

\section{From albumin to IVIG through factor VIII: The three phases of the plasma industry historical development}

Over the past fifty years, the plasma industry has gone through three phases, each one characterized by a different market driver:

\section{Albumin}

From the 1950's through the late 1960's, the demand for human albumin determined the industry's plasma processing levels: the quantity of plasma processed by the fractionation plants was calculated to generate just enough albumin as was necessary to meet the market demand. Immunoglobulins were considered as by-products, and were available in large quantities that far exceeded their demand. Some of them could not be used commercially because of their low quality, and some was destroyed after sitting on the shelves for several years. Back then, the clinical potential of immunoglobulins for treating immune deficiencies had not yet been recognized. Furthermore, the purity, quality and safety of these products were much lower than the immunoglobulins available today and their administration through the intramuscular route was cumbersome and hampered their clinical efficacy.

\section{Factor VIII}

In the late 1960's, factor VIII concentrates began to be commercially available, and factor IX a few years later. These products not only changed the lives of hemophilia A and B patients but altered the industry's focus as well: from that point in time, the plasma fractionators strived to meet the growing demand for antihemophilic factor VIII concentrate. This product soon became the market driver, replacing human albumin in this role, as the volume of plasma processed was determined by the demand for plasma-derived factor VIII. In 1992, the volume of plasma available (recovered and source) for fractionation in the US was about 8 million liters. In 1982, polyvalent intravenous immune globulin (IVIG) was launched in the United States (it was already available in Germany and Japan) by Sandoz and Cutter. Sales 
climbed rapidly because the therapeutic benefits of IVIG were promptly recognized by the medical community, and duly promoted by the companies. However, factor VIII remained the market driver because of the introduction, in the mid-1980's, of virus inactivation methods aimed at avoiding the transmission of diseases, in particular HIV. The implementation of these methods significantly reduced the factor VIII manufacturing yield. As a result, a higher volume of plasma (about 50\% more) was needed to produce the same quantity of factor VIII as before. The market introduction of the first recombinant factor VIII by Baxter at the end of 1992 brought another change in the market dynamic.

\section{Polyvalent Intravenous Immune Globulin (IVIG)}

From 1993 onward, American hemophilia A patients converted quickly to recombinant factor VIII, which became the product of choice for the newborn boys with hemophilia. While the demand for plasma-derived factor VIII dropped in the United States, the fractionators were able to re-direct their sales to other markets. Recombinant factor VIII reached western Europe and Japan in 1994, further contributing to the decline of plasma-derived product in these regions. By the mid-1990's, the demand for IVIG had reached a point where it had become the market driver, taking over factor VIII in this role. The quantity of plasma collected and processed by the fractionators was no longer determined by the factor VIII requirements, but by the demand for polyvalent immune globulin. This situation still prevails today and most likely through the next two decades. Therefore, the quantity of plasma to be collected in 2015 will be derived from the predicted quantity of IVIG needed globally.

\section{IVIG Demand from 2008 to 2012 - Impact on the plasma supply}

In recent years, IVIG usage has grown significantly in such medical areas as neurology, rheumatology, nephrology, dermatology, oncology and infectious diseases, as well as allergy and immunology, in addition to the continuing increase of the number of patients with primary immune deficiencies. Based on this usage pattern which characterizes the markets in most industrialized countries, the volume of IVIG is forecast to grow from about 82.3 metric tons in 2008 to some 107.9 tons by 2012 , corresponding to an annual growth rate of $7 \%$ - the rate observed in the past ten years. Beyond 2012, the demand of IVIG will depend upon the results of the Alzheimer's disease trials and the possible approval of IVIG for this new indication.

In 2007, some 26.5 million liters of plasma were fractionated globally (8.6 million liters of recovered plasma, and 17.9 million liters of source plasma). As this quantity generated some 75.2 metric tons of IVIG, the average production yield, on a global basis, was 2.8 grams per liter. This relatively low yield was attributed to the fact that IVIG was not manufactured by all the fractionators, or is manufactured by some old plants with low yields from plasma. 
As the yields are expected to improve over time, reaching 3.43 grams per liter by 2012, 31.7 million liters of plasma (9.2 million liters of recovered plasma, and 22.5 million liters of source) will generate 107.9 tons of IVIG that year.

In order to process this additional volume of 5.15 million liters of plasma the global fractionation capacity will have to be increased accordingly. This will be achieved by building new fractionation plants and expanding existing ones, in addition to the improvement in production yield. Several fractionators (Baxter, CSL Behring, Grifols, Kedrion and Octapharma have already announced plans to increase their fractionation capacity. The additional 5.15 million liters will consist of 0.57 million liters of recovered plasma and 4.6 million liters of source plasma, including 3.7 million liters to be collected in the United States.

Table 1

Global IVIG demand from 2006 to 2015 (Metric tons)

\begin{tabular}{|c|c|c|c|c|c|c|c|c|c|c|}
\hline Year & 2006 & 2007 & 2008 & 2009 & 2010 & 2011 & 2012 & 2013 & 2014 & 2015 \\
\hline \multicolumn{11}{|c|}{ IVIG is approved for Alzheimer's disease treatment in 2012} \\
\hline Metric tons & 68.0 & 75.2 & 82.3 & 88.1 & 94.2 & 100.8 & 107.9 & 120.8 & 135.3 & 151.6 \\
\hline Annual growth rate & & $10.6 \%$ & $9.4 \%$ & $7.0 \%$ & $7.0 \%$ & $7.0 \%$ & $7.0 \%$ & $12.0 \%$ & $12.0 \%$ & $12.0 \%$ \\
\hline \multicolumn{11}{|c|}{ IVIG is not approved for Alzheimer's disease treatment in 2012} \\
\hline Metric tons & 68.0 & 75.2 & 82.3 & 88.1 & 94.2 & 100.8 & 107.9 & 115.4 & 123.5 & 132.2 \\
\hline Annual growth rate & & $10.6 \%$ & $9.4 \%$ & $7.0 \%$ & $7.0 \%$ & $7.0 \%$ & $7.0 \%$ & $7.0 \%$ & $7.0 \%$ & $7.0 \%$ \\
\hline
\end{tabular}

The Marketing Research Bueau, Inc.

\section{IVIG demand beyond 2012 - Impact of the clinical trial for Alzheimer's disease Scenario A: IVIG is not approved for Alzheimer's' disease treatment}

If IVIG is not approved for Alzheimer's disease treatment by 2012, the global demand for IVIG will continue at the same rate of increase as before, about $7 \%$ per year, driven by the existing indications, and reach 132.3 tons by 2015 . Using a yield of 3.91 grams per liter of plasma, this demand level will require 33.8 million liters of plasma (24.4 million of source, including 17.5 million in the US and 9.5 million of recovered). Source plasma collections, both in the US and Europe, will climb by $8 \%$ between 2012 and 2015 . The gain in IVIG output will come both from yield improvement - including the coming on line of new plants - and additional plasma collections.

\section{Scenario B: IVIG is approved for Alzheimer's' disease treatment}

\subsection{IVIG and Alzheimer's disease: Rationale, clinical trials, patient population}

Five years ago, Baxter and subsequently Octapharma, initiated clinical trials to determine whether IVIG could delay the progression of Alzheimer's disease in mild 
GLOBAL DEMAND FOR POLYVALENT INTRAVENOUS IMMUNE GLOBULIN (IVIG) WITH/WITHOUT ALZHEIMER TREATMENT APPROVAL $2006-2016$

(Metric Tons)

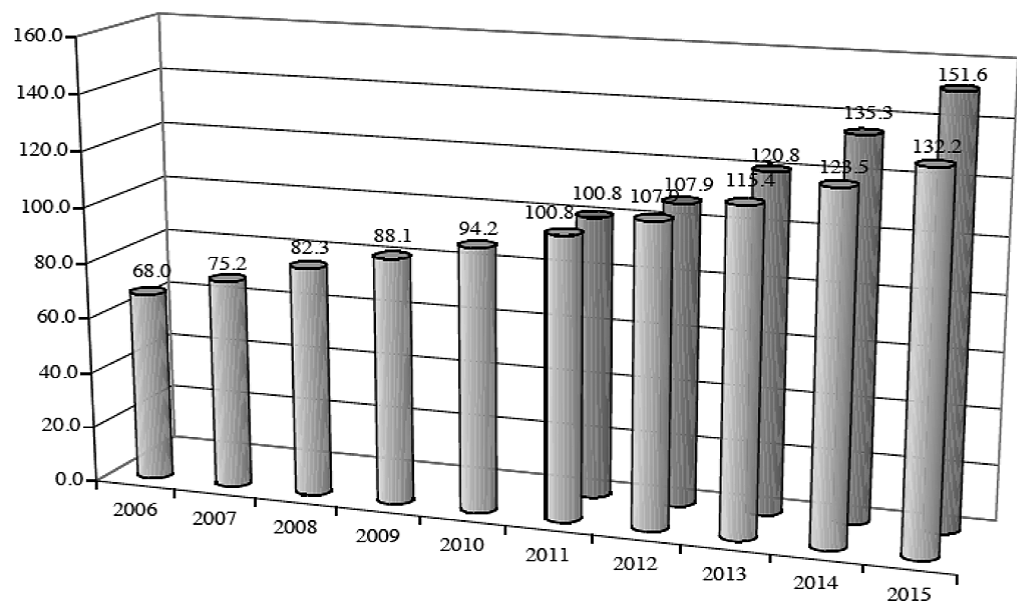

QAlzheimer not Approved $\quad$ QAlzheimer Approved

The Marketing Research Bureau, Inc.

Fig. 1.

and moderate patients. The treatment rationale relies in part on IVIG's ability to reduce the formation of beta-amyloid oligomers, thereby inhibiting the growth of senile plaques in the brain, and thus slowing down the disease process. Baxter's phase III trial began in 2009, with 360 patients enrolled. Results are expected by 2011.

The prevalence of Alzheimer's disease is unclear, in part due to wide disparities in diagnosis rates by socioeconomic class and place of residence. While the generally accepted prevalence level is between 2.4 million and 5.2 million people, a recent population survey (the ADAMS study) has suggested that the lower end of this range (2.4 million) was more likely. In Germany, it is estimated that there are some 650,000 Alzheimer's disease patients. Severe patients represent $30 \%$ of the patient population, moderate patients, $40 \%$, and mild patients, $30 \%$

$\begin{array}{cr}\text { US Alzheimer's disease patients population by age groups } \\ \text { Age } & \text { Patients } \\ 71-79 & 340,000 \\ 80-89 & 1,500,000 \\ 90^{+} & 560,000 \\ \text { Total US } & 2,400,000\end{array}$


To forecast the potential impact of IVIG on the treatment of Alzheimer's disease, the following assumptions have been made.

Table 2

IVIG is not approved for Alzheimer's disease treatment in 2012

Volume of Plasma collected Recovered

Liters x 1000

\begin{tabular}{lrrrrrr}
\hline Region & 2000 & 2005 & 2007 & 2010 & 2012 & 2015 \\
\hline Asia \& Oceania & 1,255 & 921 & 1,321 & 1,374 & 1,429 & $1,486+4 \%$ growth in 3 years \\
Latin America & 367 & 359 & 475 & 493 & 513 & $534+4 \%$ growth in 3 years \\
North America & 2,255 & 2,257 & 2,335 & 2,405 & 2,477 & $2,552+3 \%$ growth in 3 years \\
Middle East/Africa & 186 & 228 & 230 & 239 & 249 & $259+4 \%$ growth in 3 years \\
Europe & 5,150 & 3,926 & 4,238 & 4,365 & 4,496 & $4,631+3 \%$ growth in 3 years \\
World & 13,179 & 7,690 & 8,599 & 8,877 & 9,165 & 9,461 \\
\multicolumn{7}{c}{ Volume of Plasma collected Apheresis } \\
& 2000 & 2005 & 2007 & 2010 & 2012 & 2015 \\
\hline Region & 3,113 & 3,568 & 3,264 & 3,362 & 3,563 & $3,884+3 \%, 6 \%$ and 9\% growth rates \\
\hline Asia \& Oceania & 135 & 34 & 40 & 42 & 45 & $46+6 \%, 6 \%$ and 6\% growth rates \\
Latin America & 9,605 & 8,500 & 12,490 & 15,613 & 16,237 & $17,536+25 \%, 4 \%$ and 8\% growth rates \\
North America & 2 & 10 & 10 & 11 & 11 & $12+6 \%, 6 \%$ and 6\% growth rates \\
Middle East/Africa & 324 & 1,616 & 2,144 & 2,573 & 2,676 & $2,890+25 \%, 4 \%$ and 8\% growth rates \\
Europe & 9,183 & 13,728 & 17,948 & 21,600 & 22,532 & 24,369 \\
World & \multicolumn{7}{c}{ The Marketing Research Bueau, Inc. }
\end{tabular}

Table 3

IVIG is not approved for Alzheimer's disease treatment in 2012

Volume of Plasma collected - Apheresis + Recovered

\begin{tabular}{lrrrrrr}
\multicolumn{7}{c}{ Liters X 1000 } \\
\hline Region & 2000 & 2005 & 2007 & 2010 & 2012 & 2015 \\
\hline Asia \& Oceania & 4,338 & 4,489 & 4,585 & 4,736 & 4,993 & 5,371 \\
Latin America & 502 & 393 & 515 & 536 & 558 & 581 \\
North America & 11,860 & 10,757 & 14,825 & 18,018 & 18,714 & 20,087 \\
Middle East/Africa & 188 & 238 & 240 & 250 & 260 & 271 \\
Europe & 5,474 & 5,542 & 6,382 & 6,938 & 7,172 & 7,521 \\
World & 22,362 & 21,418 & 26,547 & 30,477 & 31,697 & 33,831 \\
Yield (grams per liter) & 2.12 & 3.10 & 2.83 & 3.10 & 3.41 & 3.91 \\
IVIG Production (tons) & 47.41 & 66.40 & 75.23 & 94.17 & 107.93 & 132.28 \\
\hline \multicolumn{7}{c}{ The Marketing Research Bueau, Inc. }
\end{tabular}

\subsection{Assumptions on the patient population}

a) Only patients in the 71 to 89 years age group are only considered candidates for IVIG treatment because it is believed to be more efficacious at the early stage of the disease, b) Severe patients (30\% of the total) are eliminated from the total count of IVIG treatment candidates because IVIG treatment is unlikely to be efficacious in the advance disease stage, c) Half of the potential patients are undiagnosed, and 
Table 4

IVIG is approved for Alzheimer's desease treatment in 2012

Volume of Plasma collected - Recovered Liters X 1000

\begin{tabular}{lrrrrrr}
\hline Region & 2000 & 2005 & 2007 & 2010 & 2012 & 2015 \\
\hline Asia \& Oceania & 1,225 & 921 & 1,321 & 1,374 & 1,429 & $1,486+4 \%$ growth in 3 years \\
Latin America & 367 & 359 & 475 & 493 & 513 & $534+4 \%$ growth in 3 years \\
North Ametria & 2,255 & 2,257 & 2,335 & 2,405 & 2,477 & $2,552+3 \%$ growth in 3 years \\
Middle East/Africa & 186 & 228 & 230 & 239 & 249 & $259+4 \%$ growth in 3 years \\
Europe & 5,150 & 3,926 & 4,238 & 4,365 & 4,496 & $4,631+3 \%$ growth in 3 years \\
World & 13,179 & 7,690 & 8,599 & 8,877 & 9,165 & 9,461 \\
& \multicolumn{7}{c}{ Volume of Plasma collected - Apheresis } \\
& 2000 & 2005 & 2007 & 2010 & 2012 & 2015 \\
Region & 3,113 & 3,568 & 3,264 & 3,362 & 3,563 & $3,884+3 \%, 6 \%$ and 9\% growth rates \\
Asia \& Oceania & 135 & 34 & 40 & 42 & 45 & $48+6 \%, 6 \%$ and 6\% growth rates \\
Latin America & 9,605 & 8,500 & 12,490 & 15,613 & 16,237 & $21,433+25 \%, 4 \%$ and 30\% growth rates \\
North America & 2 & 10 & 10 & 11 & 11 & $12+3 \%, 6 \%$ and 6\% growth rates \\
Middle East/Africa & 324 & 1,616 & 2,144 & 2,573 & 2,676 & $4,014+25 \%, 4 \%$ and 50\% growth rates \\
Europe & 9,183 & 13,728 & 17,948 & 21,600 & 22,532 & 29,390 \\
World & \multicolumn{7}{c}{ Liters X 1000} \\
\hline
\end{tabular}

The Marketing Research Bueau, Inc.

consequently subtracted from the total count, d) Two thirds of the remaining patients do not qualify for IVIG treatment for various socio-economic reasons (insurance coverage, access to care, lack of awareness of treatment opportunities, reluctance to use blood products, etc) e) Since the treatment efficacy remains unknown at this stage of the trials, it is assumed that only half of the patients will respond to IVIG treatment.

The number of patients actually treated with IVIG by in 2015 is therefore estimated at approximately 106,300 in the United States and 27,800 in Germany, using similar assumptions.

$\begin{array}{lr}\text { Demographic Assumptions } & \text { Patients } \\ \text { a) 71-89 age group } & 1,840,000 \\ \text { b) Mild \& moderate, representing 70\% of the above } & 1,288,000 \\ \text { c) Half of the patients are undiagnosed, representing 50\% of the above } & 644,000 \\ \text { d) Only 33\% of the above patients qualify for IVIG treatment } & 212,520 \\ \text { e) Half of the above patients do not respond to IVIG treatment } & 106,260\end{array}$

\subsection{Dosage assumptions}

Various dosage regimens have been used in the trials, ranging from 0.2 grams (Baxter trial) to 0.4 grams per kilogram bodyweight every two weeks (low dose), to 0.4 grams $/ \mathrm{Kg}$. for three days per month (Octapharma trial). The patient's weight is assumed to be 70 kilograms. Based on the above, each patient with Alzheimer's Disease will need either: 
Table 5

IVIG is approved for Alzheimer's disease treatment in 2012

Volume of Plasma collected - Apheresis + Recovered

\begin{tabular}{lrrrrrr}
\multicolumn{7}{c}{ Liters X 1000 } \\
\hline Region & 2000 & 2005 & 2007 & 2010 & 2012 & 2015 \\
\hline Asia \& Oceania & 4,338 & 4,489 & 4,585 & 4,736 & 4,993 & 5,371 \\
Latin America & 502 & 393 & 515 & 536 & 558 & 581 \\
North America & 11,860 & 10,757 & 14,825 & 18,018 & 18,714 & 23,984 \\
Middle East/Africa & 188 & 238 & 240 & 250 & 260 & 271 \\
Europe & 5,747 & 5,542 & 6,382 & 6,938 & 7,172 & 8,645 \\
World & 22,362 & 21,418 & 26,547 & 30,477 & 31,697 & 38,851 \\
Yield (grams per liter) & 2.12 & 3.10 & 2.83 & 3.10 & 3.41 & 3.91 \\
IVIG Production (tons) & 47.41 & 66.40 & 75.23 & 94.17 & 107.93 & 151.91 \\
\hline
\end{tabular}

The Marketing Research Bueau, Inc.

IVIG Treatment for Alzheimer's Disease is not approved Global Volume of Plasma Collected Source \& Recovered (Liters x 1,000

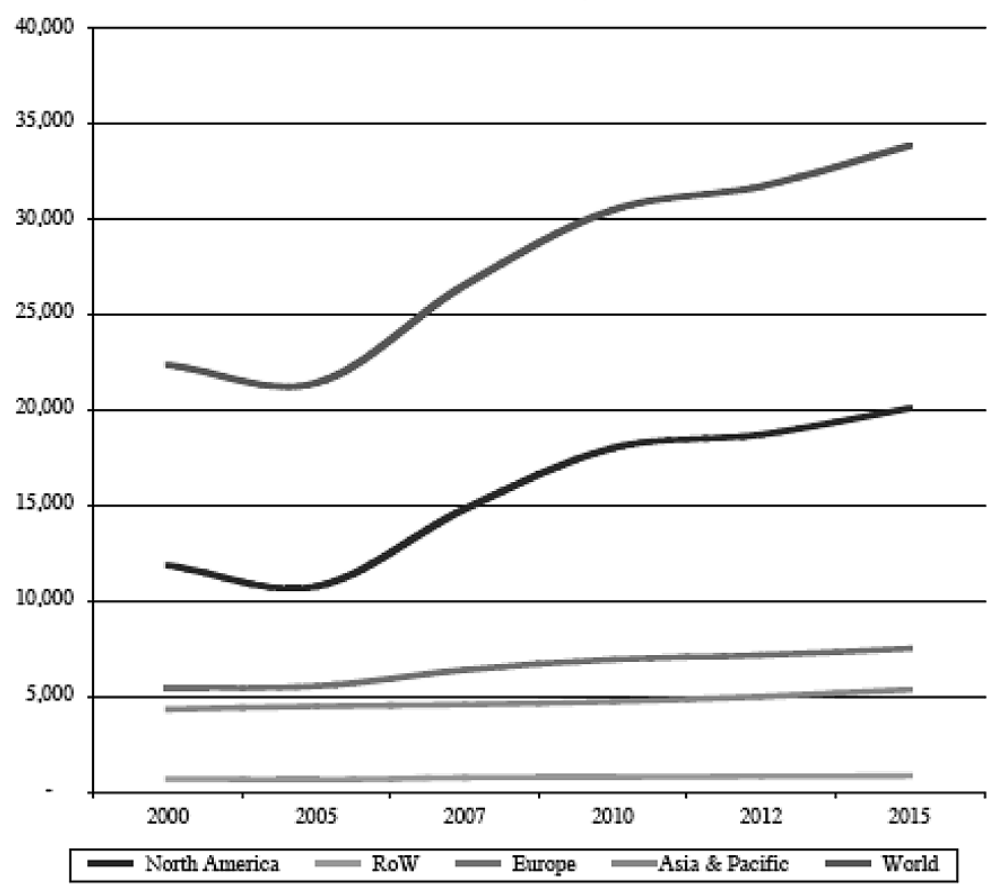

The Marketing Research Bureau, Inc.

Fig. 2. 


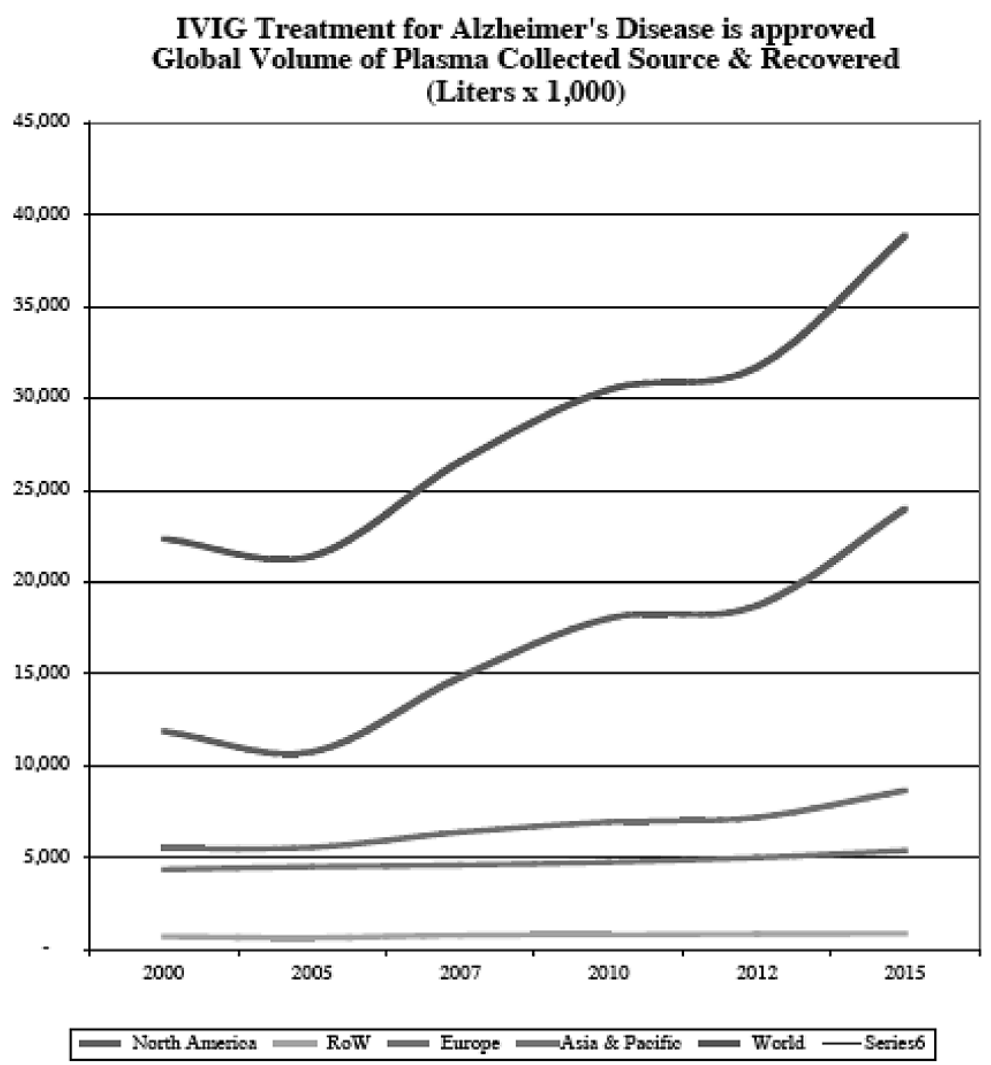

The Marketing Research Bureau, Inc.

Fig. 3.

-0.2 grams $\times 70$ kilograms $\times 26$ weeks $=364$ grams per year, or

- 0.4 grams $\times 70$ kilograms $\times 3 \times 12$ months $=1,008$ grams $/$ year

Assuming an IVIG production yield of 3.3 grams per liter, each patient will account for 110 to 305 liters of plasma per year, depending on the dosage assumption used. With a yield of 4.5 grams per liter, the plasma requirements will be 80 and 225 liters per year.

\subsection{IVIG volume requirements in 2015}

Based on the above patient populations of about 106,300 patients in the US and 27,800 in Germany, and using the low dose described above, the quantity of IVIG that will be needed to treat Alzheimer's disease patients in 2015 is approximately 48.8 
metric tons $(106,300+27,800 \times 364=48,812,000$ grams $)$ : about 38.7 tons in the US and 10.1 tons in Germany. This quantity is to be added to the 132.3 tons forecasted in 2015 , for a total of 181.1 tons. By 2015 , the fractionators will probably not be in a position to fully generate this quantity of IVIG which is equivalent to 46.3 million liters capacity (assuming a 3.91 yield per liter of plasma), a production volume which exceeds the investments in plant expansion announced by the companies - pending further expansion programs.

As the acceptance of IVIG treatment for Alzheimer's disease gradually increases, it is assumed that will add five percentage points each year to the consumption of IVIG, on top of the previous growth rate (without Alzheimer's disease treatment), going from $7 \%$ to $12 \%$ per year from 2012 onwards. Alzheimer's disease treatment with IVIG will almost certainly divert some product from its use for other indications, in particular those off-label for which the efficacy of IVIG is controversial, and not reimbursed by the insurance companies. The extent of this product diversion is hard to quantify at this stage but it is expected to constitute to better part of the off-label usage. Conversely, Alzheimer's disease treatment with IVIG is not seen as a threat to the well established conditions that are fully covered by insurance companies, such as primary immune deficiencies.

\subsection{Plasma volume and fractionation capacity requirements}

Assuming that the IVIG market will grow at the rate of $12 \%$ per year from 2013 to 2015 , the quantity of IVIG generated will be 151.6 tons in 2015 . This quantity will be obtained by adding 12.3 million liters of fractionation capacity and plasma collections worldwide between 2007 and 2015 - assuming 3.91 grams per liter production yield on an average, global basis.

\section{Acknowledgements}

The contents of this study represent our analysis of information generally available to the public or released by responsible individuals in the companies mentioned. It does not contain information provided in confidence by our clients. Since much of the information in the study is based on a variety of sources (which we deem reliable) including subjective estimates and analyst opinion, The Marketing Research Bureau, Inc. does not guarantee the accuracy of the contents and assumes no liability for inaccurate source materials. 